\title{
Some analytic results for the contribution to the anoma- lous magnetic moments of leptons due to the polarization of vacuum via lepton loops
}

\author{
Olga Solovtsova ${ }^{1,2, *}$, Vasil Lashkevich ${ }^{2}$, and Alexander Sidorov ${ }^{1}$ \\ ${ }^{1}$ BLTP, Joint Institute for Nuclear Research, Dubna, 141980, Russia \\ ${ }^{2}$ ICAS, Gomel State Technical University, Gomel, 246746, Belarus
}

\begin{abstract}
We present some new exact expressions for the contribution of the mass-dependent three-bubble diagrams to the anomalous magnetic moment of leptons $L=e, \mu$ and $\tau$. A comparison with the analytic expansions in terms of the mass ratio $m_{\ell} / m_{L}$ made in the literature, whenever relevant, is discussed.
\end{abstract}

\section{Introduction}

The anomalous magnetic moment of the charged lepton, $a_{L}=(g-2)_{L} / 2$, where $L=e, \mu$ and $\tau$, is an important object for theoretical investigations, since the measurements for both the electron and the muon have greater and greater precision. There are many excellent reviews and in-depth articles on this subject, we will limit ourselves to the recent book [1] which contains numerous references. At present, the accuracy of theoretical calculations is quite high and sufficient for comparison with experimental values for the muon and electron anomaly, $a_{\mu / e}$. (The measurement of $a_{\tau}$ value is difficult due to the very short lifetime of the tau.) At the same time, there is a long-standing deviation between the experimental measurements and theoretical predictions. The current discrepancy, $\Delta a_{L} \equiv a_{L}^{\exp }-a_{L}^{\text {theor }}$, looks as follows: $\Delta a_{\mu} \simeq(270.6 \pm 72.6) \times 10^{-11}$ for the muon [2] and $\Delta a_{e} \simeq(-87 \pm 36) \times 10^{-14}$ for the electron (see, e.g., Refs. [3, 4] for more details). The discrepancies of about $3.5 \sigma$ for the muon anomaly and of about $2.5 \sigma$ for the electron, as a rather unexpected fact that the sign of $\Delta a_{e}$ is opposite to $\Delta a_{\mu}$, give a hint of possible deviations from the Standard Model (SM).

According to the SM, the contributions to $a_{L}$ can be classified into the quantum electromagnetic (QED), hadronic and electroweak types. Currently, the calculations of the eighthand tenth-order QED contributions, which are important in finding the theoretical error, are obtained mostly by numerical integration, and a small error in the code can lead to errors in the calculations that could not be identified. Therefore, an independent determination of the QED contributions can increase the reliability of calculations.

Here we apply a technique based on the Mellin-Barnes representation, which are widely used in high energies physics (see, for example, review [5] and references therein), and we obtain analytical expressions for some eighth-order mass-dependent QED contributions corresponding to three closed lepton loops illustrated by the Feynman diagrams shown in Figs. 1-3.

\footnotetext{
*e-mail: olsol@theor.jinr.ru
} 

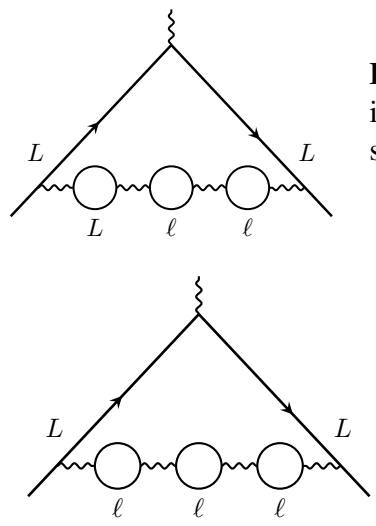

Figure 2. The diagram with three identical lepton loops are formed by leptons other than the external lepton $(\ell \neq L)$.
Figure 1. The diagram with three closed loops vacuum polarization insertion to $(g-2)_{L}$ in the case if one lepton pair is formed by the same lepton as the external one.

We use generally accepted notations (see, for example, Refs. [1, 6]) and writes the QED contribution as

$$
a_{L}^{(\mathrm{QED})}=A_{1}+A_{2}\left(\frac{m_{\ell_{1}}}{m_{L}}\right)+A_{2}\left(\frac{m_{\ell_{2}}}{m_{L}}\right)+A_{3}\left(\frac{m_{\ell_{1}}}{m_{L}}, \frac{m_{\ell_{2}}}{m_{L}}\right)
$$

where $m_{\ell_{1}}$ and $m_{\ell_{2}}$ are the masses of the leptons "inside", and $m_{L}$ is the mass of the external lepton. Each term in this sum can be expanded as a power order series in the fine structure constant $\alpha$ :

$$
A_{i}=\sum_{n \geq 1} A_{i}^{(2 n)}\left(\frac{\alpha}{\pi}\right)^{n} .
$$

The recently improved value of the fine structure constant can be found in Ref. [3].

The coefficients $A_{1}^{(2 n)}$ are universal for all leptons since corresponds the case of the closed lepton loops formed by the same leptons as the external lepton $L$, or the case where there are no lepton loops. The leading order term $A_{1}=A_{1 \text { v.p. }}^{(2)}=1 / 2$ was first obtained a long time ago by Schwinger [8]. The exact analytical results for the mass-independent coefficients $A_{1}^{(4)}$ and $A_{1}^{(6)}$ are also available [9-11] (see also Refs. [1, 12] for more details).

Among the many diagrams that contribute to the mass-dependent contribution, having the form

$$
A_{2}=A_{2}^{(2)}\left(\frac{\alpha}{\pi}\right)+A_{2}^{(4)}\left(\frac{\alpha}{\pi}\right)^{2}+A_{2}^{(6)}\left(\frac{\alpha}{\pi}\right)^{3}+A_{2}^{(8)}\left(\frac{\alpha}{\pi}\right)^{4}+\cdots,
$$

we will limit our attention to those which arise from the diagrams (the bubble diagrams) involving the lowest order vacuum polarization insertions by $\ell^{+} \ell^{-}$pairs, as an example see the diagrams shown in Figs. 1-3. The expansion coefficients in the sum (3) corresponding to this class of diagrams are denoted by the tilde, $\tilde{\mathcal{A}}_{2}^{(n)}$ (omitting v.p. in the subscript). The exact analytical result for $\tilde{\mathcal{A}}_{2}^{(4)}\left(m_{\ell} / m_{L}\right)$, corresponding to one-loop vacuum polarization insertions, was given a long time ago [13] and was reconsidered using various methods (see Refs. [14-17]). The exact analytical form for the coefficient $\tilde{\mathcal{A}}_{2}^{(6)}$ (two-loop vacuum polarization insertions) was obtained for the first time in Ref. [18], see a brief history in Ref. [19].

Our goal in this work is to present an exact analytical form for some coefficient $\tilde{\mathcal{A}}_{2}^{(8)}$ (three-loop vacuum polarization insertions). To our knowledge, the corresponding expressions have not been presented before. However, their expansions in terms of the mass ratio 
$m_{\ell} / m_{L}$ and also the numerical values $\tilde{\mathcal{A}}_{2}^{(8)}$ are well known (see the book [1] and the original publications $[6,7,20-26])$. Using the Borel transform technique in the limit when the mass ratio $m_{\ell} / m_{L}$ is small and $n \gg 1$, the coefficients $\tilde{\mathcal{A}}_{2}^{(n)}$ were obtained analytically in Ref. [27]. The analytical expressions are a serious test of both asymptotic formulas and numerous numerical results.

\section{Theoretical framework}

The idea of calculating massive Feynman integrals using a representation of the massive denominators in the form of Mellin-Barnes integrals was presented in Ref. [28] based on the following formula as the main

$$
\frac{1}{\left(k^{2}+m^{2}\right)^{\beta}}=\frac{1}{\left(k^{2}\right)^{\beta}} \frac{1}{2 \pi i} \int_{c-i \infty}^{c+i \infty} d s\left(\frac{k^{2}}{m^{2}}\right)^{-s} \frac{\Gamma(-s) \Gamma(\beta+s)}{\Gamma(\beta)} .
$$

As was shown in Ref. [16], the application of this formula and the so called converse mapping theorem [29] provides an effective way to obtain the analytic asymptotic behaviours for Feynman integrals in both the large and small ratios of mass scales.

Let us first present a simple example of using the Mellin-Barnes technique by calculation of the coefficient $\tilde{A}_{2}^{(4)}\left(m_{\ell} / m_{L}\right)$ by this method. Following Ref. [17], one can write the representation for $\tilde{\mathcal{A}}_{2}^{(4)}$ as

$$
\tilde{\mathcal{A}}_{2}^{(4)}(t)=\frac{1}{2 \pi i} \int_{c-i \infty}^{c+i \infty} d s(4 t)^{-s} \Gamma(s) \Gamma(1-s) \Omega_{0}(s),
$$

where $\left.t=m_{\ell}^{2} / m_{L}^{2}, c \in\right] 0,1\left[\right.$ is the fundamental strip, and $\Omega_{0}(s)=\frac{\Gamma(2-s) \Gamma(1+2 s)}{\Gamma(3+s)}$. This integral may be performed using the Cauchy residue theorem through residues. Then, closing the contour of integration in the complex plane to the left, we get ${ }^{1}$

$$
\begin{aligned}
& \tilde{\mathcal{A}}_{2}^{(4)}(t) \underset{t<1}{=}-\frac{25}{36}+\frac{1}{4} \pi^{2} \sqrt{t}+3 t-\frac{5}{4} \pi^{2} t^{3 / 2}+\left(\frac{44}{9}+\frac{\pi^{2}}{3}\right) t^{2}+\frac{5}{4}\left[\Phi\left(t, 2, \frac{3}{2}\right)-\frac{1}{5} \Phi\left(t, 2, \frac{5}{2}\right)\right] t^{3} \\
& -\frac{1}{6} \ln (t)+\frac{3}{2} t \ln (t)+\frac{1}{2} \sqrt{t} \operatorname{arctanh}(\sqrt{t}) \ln (t)(1-5 t)-t^{2} \ln (1-t) \ln (t)+\frac{1}{2} t^{2} \ln ^{2}(t)-t^{2} \operatorname{Li}_{2}(t),
\end{aligned}
$$

where $\Phi$ and $\mathrm{Li}_{n}$ are the Lerch function and the polylogarithm function, respectively. When closing the integration contour in Eq. (5) to the right, we get the expression

$$
\begin{aligned}
& \tilde{\mathcal{A}}_{2}^{(4)}(t) \underset{t \geq 1}{=}-\frac{1}{4}-t+\frac{1}{4 t}\left[\Phi\left(\frac{1}{t}, 2, \frac{3}{2}\right)-5 \Phi\left(\frac{1}{t}, 2, \frac{5}{2}\right)\right]-\frac{1}{6} \ln (t) \\
& +\frac{3}{2} t \ln (t)+\frac{1}{2} \sqrt{t} \operatorname{arccoth}(\sqrt{t}) \ln (t)(1-5 t)-t^{2} \ln \left(1-\frac{1}{t}\right) \ln (t)+t^{2} \operatorname{Li}_{2}\left(\frac{1}{t}\right) .
\end{aligned}
$$

The advantage of the Mellin-Barnes technique, first noted in Ref. [17], is that the resulting expressions for $t<1$ and $t>1$ are the analytic extensions of each other, and in the limit $t \rightarrow 1$, both expressions give the mass-independent result: $\tilde{\mathcal{A}}_{1}^{(4)}=\frac{119}{36}-\frac{\pi^{2}}{3}$.

\footnotetext{
${ }^{1}$ We correct a sign misprint of the second term in Ref. [17], Eq. (11).
} 
The Mellin-Barnes representation (in the notation of Ref. [21]) for $\tilde{\mathcal{A}}_{2}^{(8)}$ in the case of two identical lepton loops formed by leptons other than the external lepton' lepton $L$ (see Fig. 1) reads as

$$
\tilde{\mathcal{A}}_{2}^{(8)}\left[\text { Fig. 1] }=\frac{3}{2 \pi i} \int_{c-i \infty}^{c+i \infty} d s(4 t)^{-s} \Gamma(s) \Gamma(1-s) \Omega_{1}(s) R_{2}(s)\right.
$$

where $\Omega_{1}(s)=\Gamma(2-s)\left\{-\frac{4}{3} \frac{\Gamma(-1+2 s)}{\Gamma(1+s)}+\frac{4}{3} \frac{\Gamma(2 s)}{\Gamma(2+s)}+\frac{5}{9} \frac{\Gamma(1+2 s)}{\Gamma(3+s)}+\left[-\frac{4}{3} \frac{\Gamma(-2+2 s)}{\Gamma(s)}+2 \times\right.\right.$

$\left.\left.\frac{\Gamma(-1+2 s)}{\Gamma(1+s)}-\frac{1}{3} \frac{\Gamma(1+2 s)}{\Gamma(3+s)}\right] \mathrm{H}_{1-s}+\frac{4}{3} \frac{\Gamma(-2+2 s)}{\Gamma(s)} \mathrm{H}_{-1+s}-2 \frac{\Gamma(-1+2 s)}{\Gamma(1+s)} \mathrm{H}_{s}+\frac{1}{3} \frac{\Gamma(1+2 s)}{\Gamma(3+s)} \mathrm{H}_{2+s}\right\}$

with $\mathrm{H}_{s}=\psi(1+s)+\gamma_{\mathrm{E}}$ and $R_{2}(s)=\frac{\sqrt{\pi}}{9} \frac{(-1+s)\left(6+13 s+4 s^{2}\right)}{s^{2}(2+s)(3+s)} \frac{\Gamma(1+s)}{\Gamma\left(\frac{3}{2}+s\right)}$.

In the case of three identical lepton loops formed by leptons other than the external lepton (see Fig. 2), we use the expressions

$$
\tilde{\mathcal{A}}_{2}^{(8)}\left[\text { Fig. 2] }=\frac{1}{2 \pi i} \int_{c-i \infty}^{c+i \infty} d s(4 t)^{-s} \Gamma(s) \Gamma(1-s) \Omega_{0}(s) R_{3}(s),\right.
$$

with $R_{3}(s)=\frac{\sqrt{\pi}}{864} \frac{\Gamma(s)}{\Gamma\left(\frac{11}{2}+s\right)}\left\{\frac{P_{7}(s)}{s(1+s)(2+s)}-(1+s)\left(35+21 s+3 s^{2}\right)\left[27 \pi^{2}-162 \psi^{\prime}(s)\right]\right\}$,

and $P_{7}(s)=3492-8748 s-26575 s^{2}-9214 s^{3}+18395 s^{4}+17018 s^{5}+5120 s^{6}+512 s^{7} .^{2}$

The evaluation of the integrals (8) and (9) can be performed through residues, similar to the case of finding the coefficient $\tilde{\mathcal{A}}_{2}^{(4)}$, see Eqs. (5)-(7).

The expression for $\tilde{\mathcal{A}}_{2}^{(8)}$ [Fig. 3] has the same form as for $\tilde{\mathcal{A}}_{2}^{(8)}$ [Fig. 1] with the replacement in Eq. (8): $\Omega_{1}(s) R_{2}(s) \Rightarrow \Omega_{2}(s) R_{1}(s)$. The expression for $\Omega_{2}(s)$ is too lengthy to write here.

\section{Result and discussion}

By closing in Eq. (8) the contour of integration for $t<1$ to the left, we get the following expression:

$$
\begin{gathered}
\tilde{\mathcal{A}}_{2}^{(8)}\left[\text { Fig. 1] } \underset{t<1}{=} \frac{7627}{1944}+\frac{13 \pi^{2}}{27}-\frac{4 \pi^{4}}{45}+\left(\frac{175}{18}-\frac{4 \pi^{2}}{3}\right) t+\left(-\frac{54346}{151875}+\frac{67 \pi^{2}}{81}-\frac{8 \pi^{4}}{45}\right) t^{2}\right. \\
+\left(\frac{31168}{13505625}+\frac{2 \pi^{2}}{81}\right) t^{3}-\frac{32}{15435} t^{4}+\frac{12}{35} t^{4} \Phi\left(t, 3, \frac{9}{2}\right)-\frac{4}{45} t^{5} \Phi\left(t, 3, \frac{9}{2}\right) \\
+\frac{4}{945} t^{2}(-21+11 t) \ln ^{3}(t)+\frac{1}{27}\left(-39+108 t-67 t^{2}-2 t^{3}\right) \operatorname{Li}_{2}(t)
\end{gathered}
$$

\footnotetext{
${ }^{2}$ We fixed a typo (see Eq. (4.32) in Ref. [21]) in $\Gamma$-function argument in the denominator of Eq. (10).
} 


$$
\begin{aligned}
- & \frac{1}{3780 \sqrt{t}} \ln ^{2}(t)\left\{48(-27+7 t) \operatorname{arctanh}(\sqrt{t})+\sqrt{t}\left[-2869+420 \pi^{2}+1566 t\right.\right. \\
- & \left.\left.\left(4162-840 \pi^{2}\right) t^{2}-140 t^{3}+6\left(35+420 t-623 t^{2}+88 t^{3}\right) \ln (1-t)\right]\right\} \\
+ & \frac{1}{3} \ln ^{2}(t)\left(1+2 t^{2}\right) \mathrm{Li}_{2}(t)+\frac{1}{27}\left(9+108 t-\frac{657}{5} t^{2}+\frac{264}{35} t^{3}\right) \mathrm{Li}_{3}(t) \\
+ & \frac{1}{27} \ln (t)\left[\frac{61}{6}-\pi^{2}+\left(136-12 \pi^{2}\right) t-\left(\frac{3734}{375}-13 \pi^{2}\right) t^{2}-\frac{15936}{42875} t^{3}+\frac{48}{245} t^{4}\right. \\
+ & \frac{12}{5} t^{5} \Phi\left(t, 2, \frac{9}{2}\right)-\frac{324}{35} t^{4} \Phi\left(t, 2, \frac{9}{2}\right)+\left(-39+108 t-67 t^{2}-2 t^{3}\right) \ln (1-t) \\
+(-6 & \left.\left.-72 t+\frac{462}{5} t^{2}-\frac{264}{35} t^{3}\right) \mathrm{Li}_{2}(t)-54\left(1+2 t^{2}\right) \mathrm{Li}_{3}(t)\right]+4\left(1+2 t^{2}\right) \mathrm{Li}_{4}(t) .
\end{aligned}
$$

The accuracy currently required correlates with the uncertainty of the values of the lepton masses. Obviously, using formula Eq. (11), the numerical value of the coefficient $\tilde{\mathcal{A}}_{2}^{(8)}$ can be obtained with any accuracy. As an example, using the central mass values taken from Particle Data Group 2018 [30]: $m_{\mu}=105.6583745 \mathrm{MeV}$ and $m_{\tau}=1776.86 \mathrm{MeV}$, the numerical evaluation of the coefficient $\tilde{\mathcal{A}}_{2}^{(8)}\left[a_{\tau}^{\tau \mu \mu}\right]$ with the first 40 digits reads as: $\tilde{\mathcal{A}}_{2}^{(8)}\left[a_{\tau}^{\tau \mu \mu}\right]=0.1221159931432742475438735625265941440608 \ldots$. It interesting to note that the total eighth-order mass-dependent QED contribution up to 1100 digits of a precision was presented in Ref. [22] (see also Ref. [23]).

The exact analytic expression for the case $t>1$ looks like

$$
\begin{aligned}
\tilde{\mathcal{A}}_{2}^{(8)}[\text { Fig. 1] } & =\frac{31937}{t>1}+\frac{32}{68040}+\frac{23104}{8505 t}+\frac{6509}{630} t-\frac{334}{945} t^{2}+\frac{12}{35 t^{3}} \Phi\left(\frac{1}{t}, 3, \frac{5}{2}\right)-\frac{4}{45 t^{2}} \Phi\left(\frac{1}{t}, 3, \frac{5}{2}\right) \\
& -\frac{1}{27}\left(-39+108 t-67 t^{2}-2 t^{3}\right) \operatorname{Li}_{2}\left(\frac{1}{t}\right)-\frac{1}{3780 \sqrt{t}} \ln ^{2}(t) \\
& \times\left\{48(-27+7 t) \operatorname{arctanh}\left(\frac{1}{\sqrt{t}}\right)+\sqrt{t}\left[-139-5994 t+528 t^{2}\right.\right. \\
& \left.\left.+6\left(35+420 t-623 t^{2}+88 t^{3}\right) \ln \left(1-\frac{1}{t}\right)\right]+1260 \sqrt{t}\left(1+2 t^{2}\right) \operatorname{Li}_{2}\left(\frac{1}{t}\right)\right\} \\
& -\frac{1}{27}\left(-9-108 t+\frac{657}{5} t^{2}-\frac{264}{35} t^{3}\right) \operatorname{Li}_{3}\left(\frac{1}{t}\right)-\frac{1}{5670 t^{2}} \ln (t) \\
& \times-864-\frac{1944}{t} \Phi\left(\frac{1}{t}, 2, \frac{5}{2}\right)+504 \Phi\left(\frac{1}{t}, 2, \frac{5}{2}\right)+t\left(-7552-7895 t-27408 t^{2}\right. \\
& +2004 t^{3}+8190 t \ln \left(1-\frac{1}{t}\right)-22680 t^{2} \ln \left(1-\frac{1}{t}\right)+14070 t^{3} \ln \left(1-\frac{1}{t}\right) \\
& +420 t^{4} \ln \left(1-\frac{1}{t}\right)-36 t\left(35+420 t-539 t^{2}+44 t^{3}\right) \operatorname{Li}_{2}\left(\frac{1}{t}\right) \\
& \left.\left.+11340\left(t+2 t^{3}\right) \operatorname{Li}_{3}\left(\frac{1}{t}\right)\right)\right]-4\left(1+2 t^{2}\right) \operatorname{Li}_{4}\left(\frac{1}{t}\right)
\end{aligned}
$$


As an illustration of using Eq. (12), we give value of the coefficient $\tilde{\mathcal{A}}_{2}^{(8)}\left[a_{\mu}^{\mu \tau \tau}\right]$ with the first 40 digits: $\tilde{\mathcal{A}}_{2}^{(8)}\left[a_{\mu}^{\mu \tau \tau}\right]=8.756094385650765987299278898441049132456 \times 10^{-7}$.

In the limit $t \rightarrow 1$, both expressions (11) and (12) give the same mass-independent result $\tilde{\mathcal{A}}_{2}^{(8)}\left[\right.$ Fig. 1] $=3 \tilde{a}_{\text {univ. }}^{(8)}$, where $\tilde{a}_{\text {univ. }}^{(8)}=\frac{151849}{40824}-\frac{2 \pi^{4}}{45}+\frac{32 \zeta(3)}{63}$ (see Refs. $[1,21,31]$ ).

Let us discuss the comparison with the corresponding analytical expansions known in the literature. The expressions for $\tilde{\mathcal{A}}_{2}^{(8)}$ [Fig. 1], $\tilde{\mathcal{A}}_{2}^{(8)}$ [Fig. 2] and $\tilde{\mathcal{A}}_{2}^{(8)}$ [Fig. 3], which we get for the case $t<1$, are consistent with the corresponding expressions in Ref. [21] (see Eqs. (A1), (A3), and (A5)) and in Ref. [24] (see Eqs. for $A_{2}^{(8), \mathrm{I}(a 0)}, A_{2}^{(8), \mathrm{I}(a 1)}$, and $A_{2}^{(8), \mathrm{I}(a 2)}$ ). We do not give these expansions here but depict them as a dotted curves in Figs. 4-6 below. For the case $t>1$ we get

$$
\begin{aligned}
& \tilde{\mathcal{A}}_{2}^{(8)}\left[\text { Fig. 1] } \underset{t>1}{=}\left(\frac{5809}{1080000}-\frac{61}{54000} \ln (t)+\frac{1}{450} \ln ^{2}(t)\right) \frac{1}{t^{2}}\right. \\
& +\left(\frac{1862387}{277830000}-\frac{6073}{992250} \ln (t)+\frac{1}{350} \ln ^{2}(t)\right) \frac{1}{t^{3}} \\
& +\left[\left(\frac{12916049}{9001692000}-\frac{1940611}{200037600} \ln (t)+\frac{671}{317520} \ln ^{2}(t)\right) \frac{1}{t^{4}}+\right. \\
& \left(-\frac{15372207553}{31062505320000}-\frac{3811267}{373527000} \ln (t)+\frac{361}{242550} \ln ^{2}(t)\right) \frac{1}{t^{5}}+O\left(\frac{1}{t^{6}}\right) \text {. } \\
& \tilde{\mathcal{A}}_{2}^{(8)}\left[\text { Fig. 2] } \underset{t>1}{=}\left(\frac{87709}{9729720}-\frac{89}{15015} \zeta(3)\right) \frac{1}{t^{2}}+\left(\frac{12204667}{1824322500}-\frac{2}{1125} \ln (t)\right.\right. \\
& \left.-\frac{40}{9009} \zeta(3)\right) \frac{1}{t^{3}}+\left(\frac{73879547}{17721990000}-\frac{1}{375} \ln (t)-\frac{334}{109395} \zeta(3)\right) \frac{1}{t^{4}} \\
& +\left(\frac{26870639}{10266151896}-\frac{193}{66150} \ln (t)-\frac{688}{323323} \zeta(3)\right) \frac{1}{t^{5}}+O\left(\frac{1}{t^{6}}\right) \text {. } \\
& \left.+\frac{1023526159}{5186160000}-\frac{17}{105} \zeta(3)\right) \frac{1}{t^{2}}-\left(\frac{1243103}{187535250} \ln (t)-\frac{1061}{595350} \ln ^{2}(t)+\frac{2}{2835} \ln ^{3}(t)\right. \\
& \left.-\frac{8}{945} \zeta(3)+\frac{4744350631}{472588830000}\right) \frac{1}{t^{3}}-\left(\frac{1013327141}{199687534200} \ln (t)-\frac{166657}{57629880} \ln ^{2}(t)+\frac{2}{6237} \ln ^{3}(t)\right. \\
& \left.-\frac{8}{2079} \zeta(3)+\frac{8721404003611}{2767669224012000}\right) \frac{1}{t^{4}}-\frac{977387988901}{182797296932250} \ln (t)-\frac{4914517}{2029052025} \ln ^{2}(t) \\
& +\left(\frac{16}{135135} \ln ^{3}(t)+\frac{23356591160482123}{16468208480626402500}-\frac{64 \zeta(3)}{45045}\right) \frac{1}{t^{5}}+O\left(\frac{1}{t^{6}}\right) .
\end{aligned}
$$

Figures 4,5 , and 6 show the general behavior of the coefficient $\tilde{\mathcal{A}}_{2}^{(8)}\left(m_{\ell} / m_{L}\right)$ corresponding to the diagrams presented in Figs. 1, 2, and 3, respectably. The solid line is the exact result, the dotted line is the result of using the corresponding expansion for the case $m_{\ell} / m_{L}<1$, and the short-dashed line - for the case $m_{\ell} / m_{L}>1$, see formulas (13) - (15). 


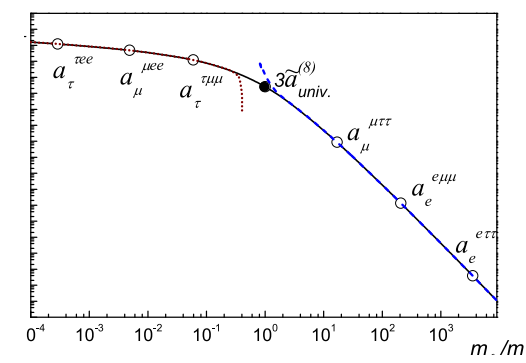

Figure 4. The behavior of the coefficient $\tilde{\mathcal{A}}_{2}^{(8)}\left[\right.$ Fig. 1] vs. the mass ratio $m_{\ell} / m_{L}$.

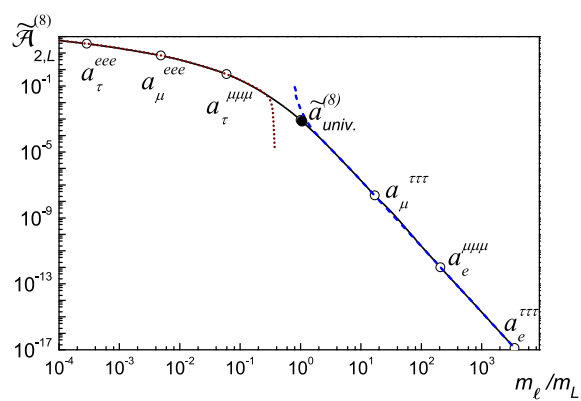

Figure 5. The behavior of $\tilde{\mathcal{A}}_{2}^{(8)}$ [Fig. 2].

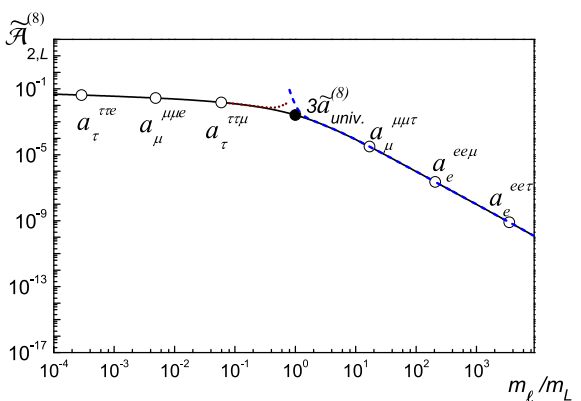

Figure 6. The behavior of $\tilde{\mathcal{A}}_{2}^{(8)}[$ Fig. 3].

Open circles indicate $\tilde{\mathcal{A}}_{2}^{(8)}\left(m_{\ell} / m_{L}\right)$ values for the physical masses of leptons. Nearby standing the designation $a$ shows the leptons that determine the contribution $\tilde{\mathcal{A}}_{2}^{(8)}(\alpha / \pi)^{4}$. The black circle corresponds to the universal value $\tilde{\mathcal{A}}_{2}^{(8)}(t=1)$. Note that expressions (11) and (12) give identical numerical values for complex $t$. For example, for $t=1+i$ the result is $\tilde{\mathcal{A}}_{2}^{(8)}[$ Fig. 1$]=0.001198244283807 \ldots-i \cdot 0.001457321196094 \ldots$. This numerically confirms that formulas (11) and (12) describe the same analytic function in the space of complex $t$.

\section{Summary}

We have presented the analytic expressions that are true for any mass ratio of the leptons for eighth order mass-dependent coefficients $\tilde{\mathcal{A}}_{2}^{(8)}(t)$ to determine the contributions to anomalous magnetic moment of leptons of the diagrams shown in Figs. 1-3. We have demonstrated numerically that exact expressions for $\tilde{\mathcal{A}}_{2}^{(8)}(t)$ are described by the same analytic function. The analytical expressions found for these coefficients will independently verify the accuracy of the result of numerical calculations. The asymptotic expressions $(t>1)$ are given that possess the necessary accuracy and are not as cumbersome as exact formulas.

\section{Acknowledgments}

We are pleased to thank the organizers of the International Workshop on High Energy Physics and Quantum Field Theory (QFTHEP'2019) for a very productive and enjoyable workshop. We are grateful to A.E. Dorokhov for interest in the work and helpful discussions and to N. V. Simaitis for technical help. The work was partially financially supported by Belarusian Republican Foundation for Fundamental Research (grant No. F18D-002) 


\section{References}

[1] F. Jegerlehner, The anomalous magnetic moment of the muon, Springer Tracts Mod.Phys. 274 (Springer International Publishing AG, 2017), 693 p.

[2] A. Keshavarzi, D. Nomura and T. Teubner, Phys. Rev. D 97, 114025 (2018).

[3] R. H. Parker, C. Yu, W. Zhong, B. Estey, and H. Mueller, Science 360, 191 (2018).

[4] H. Davoudiasl and W. J. Marciano, Phys. Rev. D 98, 075011 (2018).

[5] A. V. Kotikov and S. Teber, Phys. Part. Nucl. 50, 1 (2019).

[6] T. Kinoshita, B. Nizic, and Y. Okamoto, Phys. Rev. D 41, 593 (1990).

[7] T. Kinoshita and M. Nio, Phys. Rev. D 73, 013003 (2006).

[8] J. S. Schwinger, Phys. Rev. 73, 416 (1948) and 76, 790 (1949).

[9] A. Petermann, Helv. Phys. Acta 30, 407 (1957).

[10] C. M. Sommerfield, Phys. Rev. 107, 328 (1957).

[11] S. Laporta and E. Remiddi, Phys. Lett. B 379, 283 (1996).

[12] S. Laporta, J. Phys. Conf. Ser. 1085, no. 2, 022004 (2018).

[13] H. H. Elend, Phys. Letts. 20, 684 (1966); Errata: 21, 720 (1966).

[14] G. Li, R. Mendel and M. A. Samuel, Phys. Rev. D 47, 1723 (1993).

[15] A. Czarnecki and M. Skrzypek, Phys. Lett. B 449, 354 (1999).

[16] S. Friot, D. Greynat and E. De Rafael, Phys. Lett. B 628, 73 (2005).

[17] S. Friot and D. Greynat, J. Math. Phys. 53, 023508 (2012).

[18] S. Laporta, Nuovo Cim. A 106, 675 (1993).

[19] S. Laporta, J. Phys. Conf. Ser. 1138, 012001 (2018).

[20] S. Laporta, Phys. Lett. B 312, 495 (1993).

[21] J. P. Aguilar, D. Greynat and E. de Rafael, Phys. Rev. D 77, 093010 (2008).

[22] S. Laporta, Phys. Lett. B 772, 232 (2017).

[23] A. Kurz, T. Liu, P. Marquard and M. Steinhauser, Nucl. Phys. B 879, 1 (2014).

[24] A. Kurz, T. Liu, P. Marquard, A. Smirnov, V. Smirnov and M. Steinhauser, Phys. Rev. D 93, 053017 (2016).

[25] P. A. Baikov, A. Maier and P. Marquard, Nucl. Phys. B 877, 647 (2013).

[26] P. Marquard, A. V. Smirnov, V. A. Smirnov, M. Steinhauser and D. Wellmann, EPJ Web Conf. 218, 01004 (2019).

[27] M. L. Laursen and M. A. Samuel, Phys. Rev. D 23, 2478 (1981).

[28] E. E. Boos and A. I. Davydychev, Theor. Math. Phys. 89, 1052 (1991) [Teor. Mat. Fiz. 89, 56 (1991)].

[29] Ph. Flajolet, X. Gourdon and Ph. Dumas, Theor. Comp. Science 144, 3 (1995).

[30] M. Tanabashi et al. [Particle Data Group], Review of Particle Physics, Phys. Rev. D 98, 010001 (2018) and 2019 update.

[31] A. V. Sidorov, V. I. Lashkevich and O. P. Solovtsova, Nonlin. Phenom. Complex Syst. 21, 395 (2018). 